\title{
Backward dilepton production in color dipole and parton models
}

\author{
Maria Beatriz Gay Ducati* and Emmanuel Gräve de Oliveira ${ }^{\dagger}$ \\ Instituto de Física, Universidade Federal do Rio Grande do Sul, Caixa Postal 15051, 91501-970 Porto Alegre, \\ Rio Grande do Sul, Brazil
}

(Received 16 December 2009; published 15 March 2010)

\begin{abstract}
The Drell-Yan dilepton production at backward rapidities is studied in proton-nucleus collisions at Relativistic Heavy Ion Collider and LHC energies by comparing two different approaches: the $k_{T}$ factorization at next-to-leading order with intrinsic transverse momentum and the same process formulated in the target rest frame, i.e., the color dipole approach. Our results are expressed in terms of the ratio between $p(d)-A$ and $p-p$ collisions as a function of transverse momentum and rapidity. Three nuclear parton distribution functions are used: EKS (Eskola, Kolhinen, and Ruuskanen), EPS08, and EPS09 and, in both approaches, dileptons show sensitivity to nuclear effects, specially regarding the intrinsic transverse momentum. Also, there is room to discriminate between formalisms: the color dipole approach lacks soft effects introduced by the intrinsic $k_{T}$. Geometric scaling GBW (Golec-Biernat and Wusthoff) and BUW (Boer, Utermann, and Wessels) color dipole cross section models and also a DHJ (Dumitru, Hayashigaki, and Jalilian-Marian) model, which breaks geometric scaling, are used. No change in the ratio between collisions is observed, showing that this observable is not changed by the particular shape of the color dipole cross section. Furthermore, our $k_{T}$ factorization results are compared with color glass condensate results at forward rapidities: the results agree at Relativistic Heavy Ion Collider although disagree at LHC, mainly due to the different behavior of target gluon and quark shadowing.
\end{abstract}

DOI: 10.1103/PhysRevD.81.054015

PACS numbers: 12.38. $-\mathrm{t}, 13.85 . \mathrm{Qk}$

\section{INTRODUCTION}

Forward physics is a subject of research both at Relativistic Heavy Ion Collider (RHIC) [1] and at LHC [2] and the new experimental data offer an opportunity to improve our knowledge on how parton distribution functions (of free protons and of bound nucleons) will behave as smaller momentum fractions $x$ are reached. One of the issues is where saturation - such as given by the color glass condensate $[3,4]$-is present. When comparing nuclear collisions to proton-proton collisions, phenomena like PHENIX photon suppression [5], Cronin effect [6], and others [1,2], one can ask how nuclear effects at small $x$ can change cross sections and which modifications seen in nuclear collisions can be accounted to initial or to final state effects.

In symmetrical hadron-hadron collisions, such as proton-proton and nucleus-nucleus $(A-A)$ in the center of momentum reference frame, backward and forward rapidities give the same results for calculations of QED and QCD interactions. Therefore, the same effects observed in forward rapidities are observed at backward rapidities. However, in proton-nucleus collisions, forward rapidities imply small- $x$ effects in the nucleus, while backward rapidities imply small- $x$ effects in the proton [7]. So, at backward rapidities we can study nuclear effects and

\footnotetext{
*beatriz.gay@ufrgs.br

†emmanuel.deoliveira@ufrgs.br
}

small- $x$ physics at the same time but linked together in a complementary way. For this study, we advocate the use of dileptons as probes, since they escape untouched by the strong interactions present after a nuclear collision.

In an earlier work [7], it was demonstrated that the production of dileptons at backward rapidities could be calculated in the rest frame of the proton, taking the nucleus as the projectile and using nDS (de Florian and Sassot ) [8] or EKS (Eskola, Kolhinen, and Ruuskanen [911]) nuclear parton distribution functions and GBW (Golec-Biernat and Wusthoff [12]) color dipole cross section. Nuclear modification factors relating $p-A$ and $p-$ $p$ cross sections were calculated and showed interesting dependence in the nuclear effects, including nuclear shadowing in LHC backward predictions.

The purpose of the present work is twofold. First, we update our earlier calculations using new nuclear parton distributions functions (EPS08 [13], EPS09 [14]) and new color dipole cross sections (BUW [Boer, Utermann, and Wessels [15]], DHJ [Dumitru, Hayashigaki, and JalilianMarian [16,17]], and the new fit of GBW [18]). These $n$ PDF's show that the amplitude of nuclear effects is not well settled; in particular, the amount of shadowing present in heavy ions is not known. Following the GBW model to the color dipole cross sections, the DHJ model introduced a fit to heavy ion data and a geometric scaling breaking. Afterwards, BUW model showed that the same data can be well fitted by a geometric scaling parametrization, making it interesting to know whether dilepton production is sen- 
sitive to the breaking of geometric scaling. Second, color dipole results will be compared with the standard intrinsic $k_{T}$ approach $[19,20]$. Since most of the fitting of parton distributions is done in the parton model, it is interesting to understand the differences between the two approaches.

\section{BACKWARD DILEPTON PRODUCTION}

\section{A. Color dipole approach}

In the color dipole approach [21,22], the Drell-Yan dilepton production is studied in the rest frame of the target, in which the relevant subprocess is the scattering of a projectile parton off the target color field. The scattered parton emits a virtual photon that splits into the dilepton. The emission of the photon can happen before or after the parton interaction with the target color field, and the sum of both diagrams results in the following cross section, including the dipole cross section [22]:

$$
\frac{d \sigma}{d M^{2} d y d^{2} p_{T}}=\frac{\alpha_{\mathrm{em}}^{2}}{6 \pi^{3} M^{2}} \int_{0}^{\infty} d \rho W\left(x_{2}, \rho, p_{T}\right) \sigma_{\mathrm{dip}}\left(x_{1}, \rho\right),
$$

in which $y$ is the photon rapidity, $M$ is the dilepton mass, $p_{T}$ is the dilepton transverse momentum, $\rho$ is the dipole transverse size, and the variables $x_{1}$ and $x_{2}$ are given by

$$
x_{1,2}=\sqrt{\frac{M^{2}+p_{T}^{2}}{s}} \mathrm{e}^{ \pm y} .
$$

The weight function $W\left(x_{2}, \rho, p_{T}\right)$ depends on the projectile composition. It weights each $\rho$-sized dipole contribution to the cross section and is written as

$$
\begin{aligned}
W\left(x_{2}, \rho, p_{T}\right)= & \sum_{q} \int_{x_{2}}^{1} \frac{d \alpha}{\alpha^{2}} e_{q}^{2}\left[\frac{x_{2}}{\alpha} f_{q}^{A}\left(\frac{x_{2}}{\alpha}, M^{2}\right)+\frac{x_{2}}{\alpha} f_{\bar{q}}^{A}\right. \\
& \left.\times\left(\frac{x_{2}}{\alpha}, M^{2}\right)\right]\left\{\left[m_{q}^{2} \alpha^{4}+2 M^{2}(1-\alpha)^{2}\right]\right. \\
& \times\left[\frac{1}{p_{T}^{2}+\eta_{q}^{2}} T_{1}(\rho)-\frac{1}{4 \eta_{q}} T_{2}(\rho)\right] \\
& +\left[1+(1-\alpha)^{2}\right]\left[\frac{\eta_{q} p_{T}}{p_{T}^{2}+\eta_{q}^{2}} T_{3}(\rho)\right. \\
& \left.\left.-\frac{1}{2} T_{1}(\rho)+\frac{\eta_{q}}{4} T_{2}(\rho)\right]\right\} .
\end{aligned}
$$

In the above expression, $\eta_{q}^{2}=(1-\alpha) M^{2}+\alpha^{2} m_{q}^{2}$ and the functions $T_{i}$ are given by products of Bessel functions

$$
\begin{aligned}
& T_{1}(\rho)=\frac{\rho}{\alpha} J_{0}\left(\frac{p_{T} \rho}{\alpha}\right) K_{0}\left(\frac{\eta \rho}{\alpha}\right) \\
& T_{2}(\rho)=\frac{\rho^{2}}{\alpha^{2}} J_{0}\left(\frac{p_{T} \rho}{\alpha}\right) K_{1}\left(\frac{\eta \rho}{\alpha}\right) \\
& T_{3}(\rho)=\frac{\rho}{\alpha} J_{1}\left(\frac{p_{T} \rho}{\alpha}\right) K_{1}\left(\frac{\eta \rho}{\alpha}\right) .
\end{aligned}
$$

As in our earlier work [7], we considered the proton as the target and the nucleus as the projectile to obtain dileptons at backward rapidities. Therefore, the approach used here is phenomenologically valid for small $x_{1}$ [22], insofar as to guarantee that the parton-photon fluctuation lifetime is much larger than the parton-color field interaction time. In what was presented in this framework, $x_{2} / A$ is the projectile momentum fraction carried by photon, while $x_{2} / A / \alpha>x_{2} / A$ is carried by the projectile parton and $\alpha$ is the parton momentum fraction carried by the photon. The present work and the one of Ref. [7] differ on that, in the latter, all quarks masses were $0.2 \mathrm{GeV}$, while now the quark masses given by the color dipole parametrizations are taken into account, besides considering heavy quarks contributions.

In the above equations, the nuclear parton distribution functions (nPDF) $f_{q}^{A}$ and the dipole cross section $\sigma_{\text {dip }}\left(x_{1}, \rho\right)$ will be parametrized by fits described below. Whereas the nPDF accounts for the parton distribution in the projectile, the dipole cross section represents the interaction of the emitted parton and the target. The dipole cross section is the same as the deep inelastic scattering and includes in its fit nonperturbative effects. To study $p-p$ collisions, one has simply to change nPDFs by free proton PDFs. It is important to highlight that the color dipole approach is a parameter-free description; it only uses phenomenological parametrizations that are universal, i.e., they are the same as the ones used in deep inelastic scattering (DIS) and other processes.

\section{B. Improved parton model}

In this section, we are representing the same process studied in the last section but on the standard infinite momentum frame, using the $k_{T}$ factorization at next-toleading order (NLO). This way to study the dilepton production is also called the (improved) parton model (IPM) and was used by Drell and Yan [23,24]. In this frame, the process is understood as the combination of two partons to create the virtual photon that is going to split into the dilepton (we do not sum the $Z$ contribution, since we are working with dileptons of mass lower than $Z$.) If partons are considered collinear to hadrons, experimental results of $p_{T}$ distribution cannot be reproduced for small $p_{T}$. This problem is fixed if one introduces (two-dimensional) parton intrinsic transverse momentum $[19,20]$. The cross section at NLO is given by $[22,25]$

$$
\begin{aligned}
\frac{d \sigma}{d M^{2} d y d^{2} p_{T}}= & h\left(p_{T}^{2}\right) \frac{d \sigma}{d M^{2} d y}+\int d^{2} k_{T} \sigma_{P}\left(s, M^{2}, k_{T}^{2}\right) \\
& \times\left[h\left(\left(\mathbf{p}_{T}-\mathbf{k}_{T}\right)^{2}\right)-h\left(p_{T}^{2}\right)\right] .
\end{aligned}
$$

The intrinsic $k_{T}$ distribution of a single parton is given by $\frac{1}{\pi\left\langle k_{T}^{2}\right\rangle} \exp \left(-\frac{k_{T}^{2}}{\left\langle k_{T}^{2}\right\rangle}\right)$, with $\left\langle k_{T}^{2}\right\rangle=\frac{4}{\pi}\left\langle k_{T}\right\rangle^{2}$, and its standard deviation is $\sqrt{\frac{2}{\pi}}\left\langle k_{T}\right\rangle$. The Gaussian distribution of intrinsic $k_{T}$ 
due to both partons given by $h\left(k_{T}^{2}\right)=\frac{1}{2 \pi\left\langle k_{T}^{2}\right\rangle} \exp \left(-\frac{k_{T}^{2}}{2\left\langle k_{T}^{2}\right\rangle}\right)$ In an NLO study of pion production [26], $\left\langle k_{T}^{2}\right\rangle=2.5 \mathrm{GeV}$ was found to reproduce RHIC data even for low $p_{T}$.

The right-hand side of Eq. (4) has two terms: the first (second) term is dominant at small (high) $p_{T} /\left\langle k_{T}\right\rangle$. In the first term we have the NLO collinear double differential cross section $d \sigma / d M^{2} d y$, which shows no $p_{T}$ dependence and, therefore, this first term shows a factorized Gaussian dependence on $p_{T}$. Only noncollinear subprocesses contribute to the second term of the right-hand side of Eq. (4), meaning, Compton scattering $q+g \rightarrow q+\gamma^{*}$ and annihilation $q+\bar{q} \rightarrow g+\gamma^{*}$. To the effect of the calculation of these subprocesses, partons do not have transverse momentum; this is included in the product in Eq. (4). We have [25]

$$
\begin{aligned}
\sigma_{P}\left(s, M^{2}, p_{T}^{2}\right)= & \frac{1}{\pi^{2}} \frac{\alpha^{2} \alpha_{s}}{M^{2} \hat{s}^{2}} \int_{x_{A_{\min }}}^{1} d x_{A} \frac{x_{B} x_{A}}{x_{A}-x_{1}} \\
& \times\left\{P_{q \bar{q}}\left(x_{A}, x_{B}, M^{2}\right) \frac{8}{27} \frac{2 M^{2} \hat{s}+\hat{u}^{2}+\hat{t}^{2}}{\hat{t} \hat{u}}\right. \\
& +P_{q g}\left(x_{A}, x_{B}, M^{2}\right) \frac{1}{9} \frac{2 M^{2} \hat{u}+\hat{s}^{2}+\hat{t}^{2}}{-\hat{s} \hat{t}} \\
& \left.+P_{g q}\left(x_{A}, x_{B}, M^{2}\right) \frac{1}{9} \frac{2 M^{2} \hat{t}+\hat{s}^{2}+\hat{u}^{2}}{-\hat{s} \hat{u}}\right\},
\end{aligned}
$$

in which the subprocess Mandelstam variables are given by: $\hat{s}=x_{A} x_{B} s, \hat{t}=M^{2}-x_{A} x_{2} s$, and $\hat{u}=M^{2}-x_{B} x_{1} s$; with parton momentum fractions represented by $x_{A}$ and $x_{B}$. The integration lower limit is $x_{A_{\min }}=\left(x_{1}-\right.$
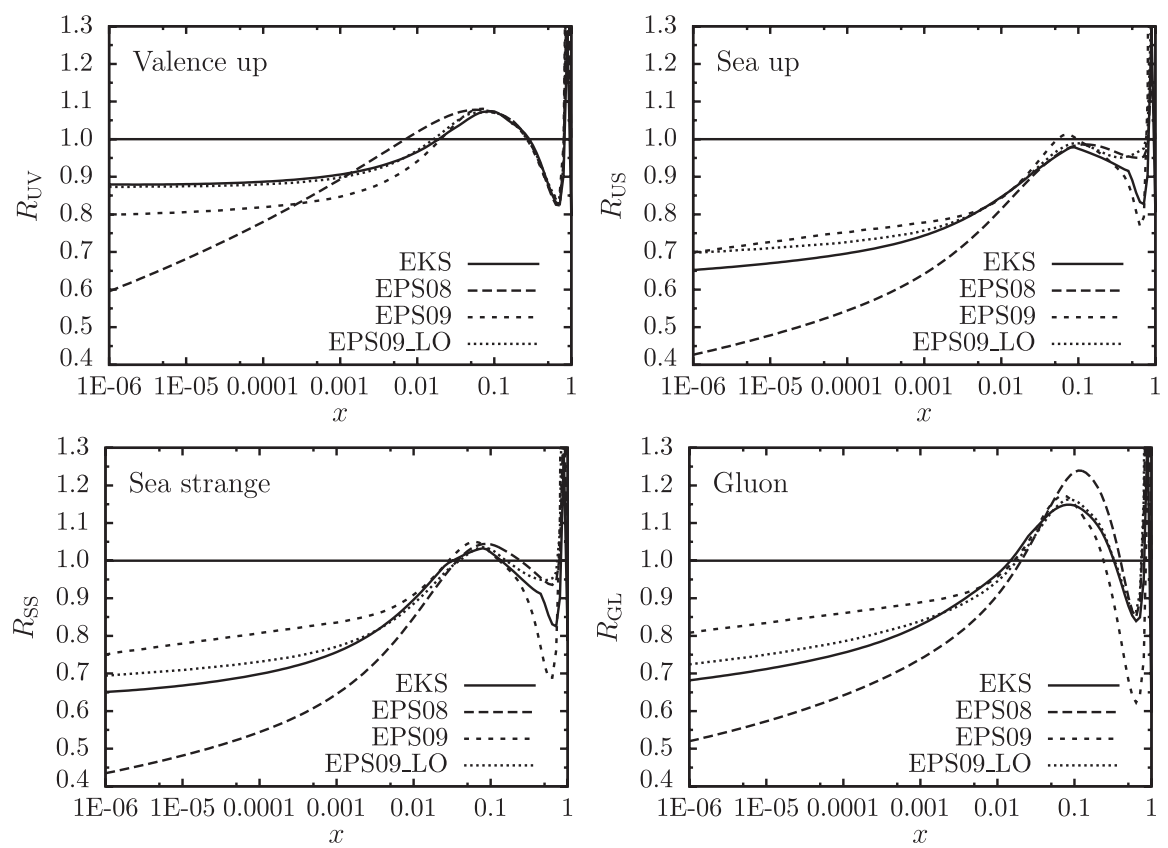
$\left.x_{1}\right)$, and are used for the $B$ hadron.

\section{Parametrizations} is

FIG. 1. EKS, EPS08, and EPS09 nuclear modification factors $R_{q}^{A}(x, Q)$ with $A=197$ and $Q=6.5 \mathrm{GeV}$ and flavors valence up (UV), sea up (US), sea strange (SS), and gluon (GL).

$\left.M^{2} / s\right) /\left(1-x_{2}\right), x_{B}$ is given by $\left(x_{A} x_{2}-M^{2} / s\right) /\left(x_{A}-\right.$

$$
\begin{aligned}
& P_{q \bar{q}}\left(x_{A}, x_{B}\right)=\sum_{q} e_{q}^{2}\left(f_{q}\left(x_{A}\right) f_{\bar{q}}\left(x_{B}\right)+\bar{q} \leftrightarrow q\right) \\
& P_{q g}\left(x_{A}, x_{B}\right)=\sum_{q} e_{q}^{2}\left(f_{q}\left(x_{A}\right)+f_{\bar{q}}\left(x_{A}\right)\right) f_{g}\left(x_{B}\right) \\
& P_{g q}\left(x_{A}, x_{B}\right)=\sum_{q} e_{q}^{2} f_{g}\left(x_{A}\right)\left(f_{q}\left(x_{B}\right)+f_{\bar{q}}\left(x_{B}\right)\right),
\end{aligned}
$$

in which PDFs are needed and, when it is the case, nPDFs

Three parametrizations of the nuclear PDFs are used: EKS [9-11], EPS08 [13], and EPS09 [14], all giving the nuclear proton PDF as the free proton PDF multiplied by a factor $f_{q}^{\text {proton-A }}(x, Q)=R_{q}^{A}(x, Q) f_{q}^{p}(x, Q)$. To obtain the nuclear neutron PDF, one relies on the isospin symmetry: $f_{q}^{\text {neutron- } A}(x, Q)=R_{q^{\prime}}^{A}(x, Q) f_{q^{\prime}}^{p}(x, Q)$; where, if $q$ is up (or down), $q^{\prime}$ is down (or up). Heavier quarks $(s, c, b, t)$ neutron distributions are the same as the proton ones. For example, the normalized total up distribution in a nucleus

$$
f_{u}^{A}=\frac{Z}{A} R_{u}^{A}(x, Q) f_{u}^{p}(x, Q)+\frac{A-Z}{A} R_{d}^{A}(x, Q) f_{d}^{p}(x, Q) .
$$

The parametrizations EKS and EPS08 are available only at leading order, while EPS09 is available at both LO and NLO accuracy. In Fig. 1, we have plotted $R_{q}^{A}(x, Q)$ with 
$A=197$ and scale $Q=6.5$ of nPDFs for four parton species: valence up quark (UV), sea up quark (US), strange quark (SS), and gluon (GL). Four regions of nuclear effects can be identified: shadowing $(x \lesssim 0.01)$, antishadowing $(0.01 \lesssim x \lesssim 0.3)$, EMC effect $(0.3 \lesssim x \lesssim 1)$, and Fermi motion $(x \approx 1)$.

It should be noted that LO EPS09 looks very similar to EKS and, therefore, we do not use LO EPS09. While EKS took into account deep inelastic lepton-nucleus scattering and Drell-Yan dilepton production only, EPS08 analysis included RHIC BRAHMS inclusive high $p_{T}$ hadron production data at high rapidities that reached $x$ around $10^{-4}$. As a result, EPS08 shows a strong shadowing, which was argued in Ref. [27]. EPS09, besides going to NLO, did not consider hadron production used in EPS08; the argument being that baseline-free proton PDFs do not accurately describe this proton-proton data, therefore invalidating attempts to use this nuclear data to constraint nPDFs. EPS09 fit included RHIC PHENIX inclusive pion production at mid rapidities ( $x$ around $10^{-2}$ ) and a less strong gluon shadowing was found. As in EPS08 and EPS09 original analysis, we use CTEQ6.1 [28] as the parton distribution function (PDF) of free protons. This PDF and the nPDFs used here employ the zero-mass variable flavor number scheme, in which the heavy quarks are massless and active only when the factorization scale is greater than the mass threshold $m_{q}^{2}$.

Dipole cross section in DIS is the cross section between the color dipole component of the virtual photon and the target, which was modeled [12] as $\sigma_{\mathrm{GBW}}(x, r)=\sigma_{0}[1-$ $\left.\exp \left(-\frac{1}{4} r^{2} Q_{s}^{2}(x)\right)\right]$, with saturation scale given by $Q_{s}^{2}(x)=$ $Q_{0}^{2}\left(\frac{x_{0}}{x}\right)^{\lambda}$, with $Q_{0}^{2}$ of $1 \mathrm{GeV}^{2}$ and the other parameters are fitted. The model reproduces color transparency for small $r$ $\left(\sigma_{\text {dip }}(x, r) \propto r^{2}\right)$ and saturation for large $r\left(\sigma_{\text {dip }}(x, r) \approx\right.$ $\left.\sigma_{0}\right)$. In the original GBW fit to DIS HERA data with $x<$ 0.01 the parameters were found to be $\sigma_{0}=29.12 \mathrm{mb}$ $\left(74.78 \mathrm{GeV}^{-2}\right), x_{0}=0.41 \times 10^{-4}$, and $\lambda=0.277$. The light quark masses used were $0.14 \mathrm{GeV}$ and the charm mass was $1.5 \mathrm{GeV}$. In a recent fit [18] of the same model but with charm mass of $1.4 \mathrm{GeV}$, the parameters found were $\sigma_{0}=23.9 \mathrm{mb}\left(61.38 \mathrm{GeV}^{-2}\right), x_{0}=1.11 \times 10^{-4}$, and $\lambda=0.287$. The dipole cross section dependence on $r$ and $x$ only through the combination $r^{2} Q_{s}^{2}(x)$ means that the deep inelastic scattering cross section obeys geometric scaling [29] (at small $x$ ), i.e., depends only on the combination $Q / Q_{s}^{2}(x)$.

The recently proposed DHJ and BUW models were used to fit forward $d$-Au RHIC hadron production data using the color glass condensate (CGC) model. The DHJ model violates geometric scaling and BUW, while similar to DHJ, was an attempt to keep geometric scaling and still fit the same data. It is an important question if one can find an observable that discriminates both models in hadronhadron collisions since, in this kind of collisions, it is not possible to observe geometric scaling directly from the data (as it is possible in the case of DIS.)
In the DHJ and BUW original papers, the dipole scattering amplitudes used depend on $r, x$, and hadron transverse momentum and represented quark and gluon interactions with the medium. However, these dipole scattering amplitudes can be rewritten to represent the dipole interaction with the medium, which depends on $r, x$, and the virtual photon mass $M$ (or the photon virtuality $Q$ in the case of DIS). Both models start with the following expression:

$$
\sigma_{\text {dip }}(x, r)=\sigma_{0} N_{\gamma}=\sigma_{0}\left[1-\exp \left(-\frac{1}{4}\left(r^{2} Q_{s}^{2}\right)^{\gamma(M, x)}\right)\right] .
$$

The above dipole cross section is different from the GBW model only by the introduction of the anomalous dimension $\gamma(M, x)$ and if $\gamma(M, x)=1$, BUW and DHJ models would reduce to the GBW model, except that they use parameters $\sigma_{0}=21 \mathrm{mb}\left(53.93 \mathrm{GeV}^{-2}\right), x_{0}=3 \times 10^{-4}$, and $\lambda=0.3$.

In the DHJ model the anomalous dimension reads

$$
\gamma(M, x)=\gamma_{s}+\left(1-\gamma_{s}\right) \frac{\left|\log \left(M^{2} / Q_{s}^{2}\right)\right|}{\lambda Y+d \sqrt{Y}+\left|\log \left(M^{2} / Q_{s}^{2}\right)\right|},
$$

with $Y=\log 1 / x$ and the parameter $d=1.2$ was fitted to data. The additional dependence on $x$ not through $M^{2} / Q_{s}^{2}$ (namely, the term $\lambda Y+d \sqrt{Y}$ in the denominator) breaks the geometric scaling. The anomalous dimension value at $M^{2}=Q_{s}^{2}$ is $\gamma_{s}=0.628$ and, when $M \rightarrow \infty, \gamma(M, x)=1$. In the BUW model, the anomalous dimension does not depend on $x$ separately, but only on the variable $w=$ $\sqrt{M^{2} / Q_{s}^{2}(x)}$, and is given by

$$
\gamma(w)=\gamma_{s}+\left(1-\gamma_{s}\right) \frac{w^{a}-1}{\left(w^{a}-1\right)+b},
$$

having the best fit with $a=2.82$ and $b=168$. Again, $\gamma(1)=\gamma_{s}$ and $\gamma(w \rightarrow \infty)=1$, i.e., both limits give the same results whether DHJ or BUW anomalous dimension is used; the difference between the models is in the fitting of the intermediate values of the anomalous dimension. It was shown [15] that both DHJ and BUW models can explain RHIC results at small $x$, but at larger $x$ DHJ model

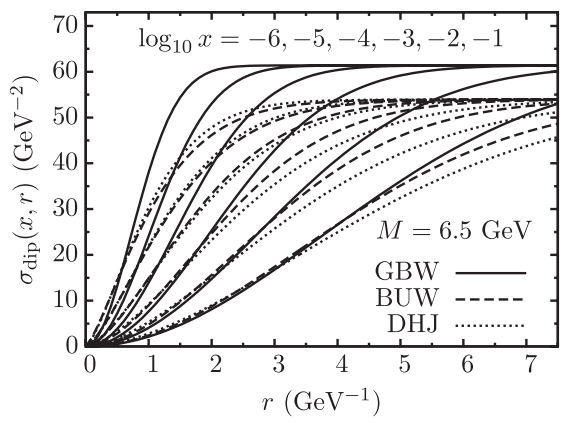

FIG. 2. Color dipole cross section as a function of dipole size $r$ and at different $x$ values. 
deviates from data. At LHC energies, in the absence of data, both models disagree even at small $x$. Thus, it is interesting to investigate whether DHJ and BUW disagree in the case of dilepton production at backward rapidities, possibly due to the breaking of geometric scaling in DHJ.

In Fig. 2, we have plotted the three models and it is seen that GBW has a bigger saturation value that plays an important role for small $x$. Also, DHJ and BUW behaviors are very similar and both models disagree most at large $x$ and mid dipole sizes.

\section{RESULTS}

The dilepton mass was fixed as $6.5 \mathrm{GeV}$ and RHIC $p-$ $p$ and $d-A u$ collisions at $200 \mathrm{GeV}$ and LHC $p-p$ and $p-P b$ collisions at $8.8 \mathrm{TeV}$ were analyzed. The results for the dilepton production cross sections in $p-p$ collisions at RHIC energies are presented in Fig. 3, where some disagreement among the $\sigma_{\text {dip }}$ is seen, specially at high $p_{T}$, when larger dipole sizes become important. Using $\left\langle k_{T}^{2}\right\rangle=$ $0.5,2.5 \mathrm{GeV}^{2}$, the effects of intrinsic $k_{T}$ are easily seen, changing the cross section behavior at small $p_{T} /\left\langle k_{T}^{2}\right\rangle$.

The nuclear modification factor is given by

$$
R_{p A}=\frac{d \sigma(p A)}{d p_{T}^{2} d y d M} / A \frac{d \sigma(p p)}{d p_{T}^{2} d y d M}
$$

In the case of $d-A$ collisions, deuteron nuclear effects are neglected and the ratio is divided by 2 times $A$ instead of only $A$. To understand how nuclear effects are taken into account in the cross sections, one has to look at the integrations of the nPDF. For example, in the color dipole approach, the Eq. (3) takes the integration of nuclear effects from $x_{2}$ to 1 . Similar dependence on $x_{2}$ can be found in the IPM. Therefore, as $x_{2}$ increases, the nuclear effects of shadowing, antishadowing, and EMC effect are sequentially dropped from calculations in this order. When shadowing is dropped it is expected that the nuclear modification factor is going to increase, since shadowing has a contribution lower than 1 . Then, when antishadowing is dropped at bigger $x_{2}$, the factor decreases. When reaching $x_{2}$ values near the antishadowing-EMC effect transition, the nuclear modification factor will grow again.

The nuclear modification factor $R_{d A}$ using EPS09 and EPS08 nPDF at RHIC energies is calculated in Fig. 4, where this ratio is given as a function of the transverse momentum at four fixed rapidities. EPS09 and EPS08 give approximately the same results since the main contribution to the ratio comes from two nuclear effects (antishadowing and EMC effect) that are described as nearly the same by both parametrizations. From $y=-1.0$ to $y=-2.5, x_{2}$ increases and antishadowing becomes less important as

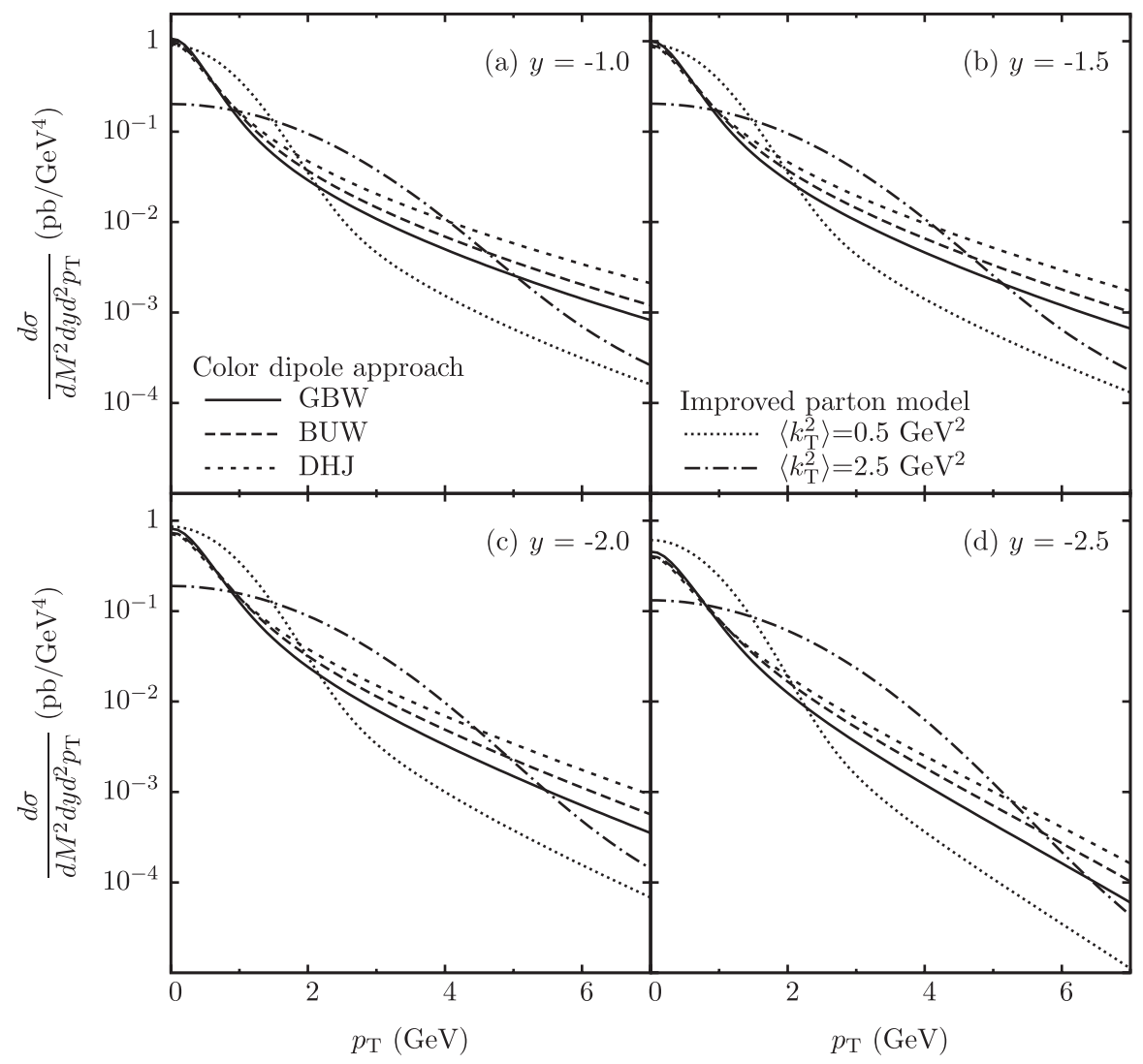

FIG. 3. Dilepton production cross sections in $p-p$ collisions at RHIC energies as a function of the transverse momentum at fixed rapidities. 


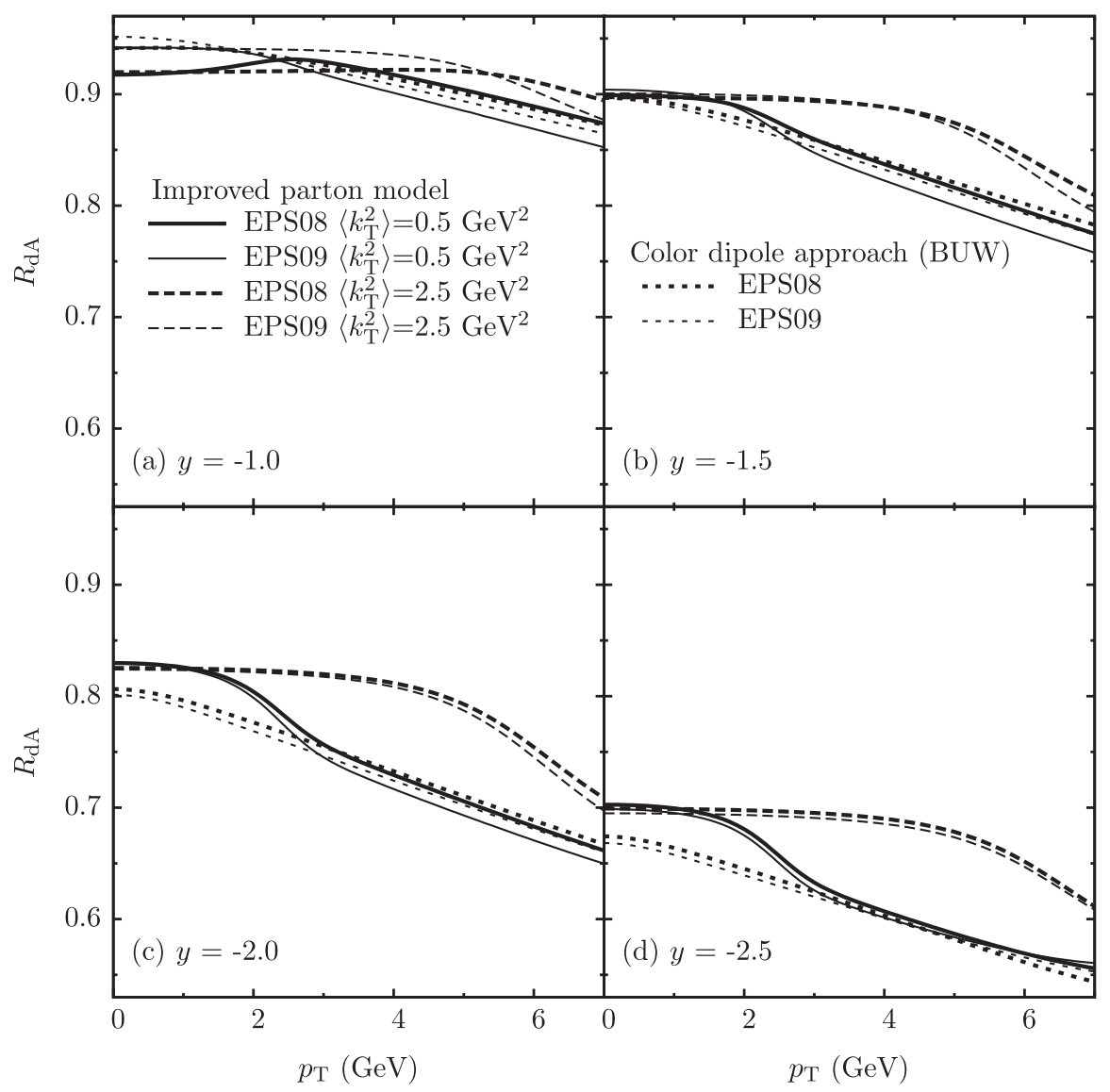

FIG. 4. Factor $R_{d A}$ at RHIC energies as a function of the transverse momentum at fixed rapidities, using EPS08 and EPS09 nPDFs.

the EMC effect dominates, driving $R_{d A}$ to lower values. The nuclear effects are very dependent on the intrinsic $k_{T}$; however, at high $p_{T} /\left\langle k_{T}\right\rangle$, dipole approach and the IPM agree, since at this point intrinsic $k_{T}$ plays a minor role because Compton scattering is the dominant subprocess. An increase in $p_{T}$ corresponds to an increase in $x_{2}$ at fixed rapidity. Therefore, the overall slope in the $p_{T}$ dependence is negative at RHIC. In Fig. 5, EPS09 and EKS are compared and both nPDFs give approximately the same results. Despite showing considerable difference in the predicted cross sections in Fig. 3, different models for $\sigma_{\text {dip }}$ provide almost identical ratios $R_{d A}$.

Based on RHIC results, it is obtained that these models and parametrizations employed agree to 5\% accuracy, apparently leading to the conclusion that dileptons at backward rapidities would not be very sensitive to different models fitted to $p-p$ results, despite that backward rapidity nuclear effects (mainly antishadowing and EMC) are involved.

The dilepton production cross sections in $p-p$ collisions at LHC energies are presented in Fig. 6, using $\left\langle k_{T}^{2}\right\rangle=$ $0.5,2.0$, and $4.5 \mathrm{GeV}^{2}$ in the IPM. Considering the different dipole cross sections, DHJ and BUW models present agreement, while disagreeing with GBW model. As it was explained in RHIC results in Fig. 3, important effects due to intrinsic $k_{T}$ can be seen and the cross sections are subject to variation as $\left\langle k_{T}\right\rangle$ and $\sigma_{\text {dip }}$ change.

In Fig. 7, $R_{p A}$ for LHC $p-\mathrm{Pb}$ collisions is given, where the interplay of the following three nuclear effects is seen: shadowing, antishadowing, and EMC effect: from $y=$ -1.5 to $y=-6.0, x_{2}$ increases and the ratio first increases (shadowing-antishadowing transition) and then decreases (antishadowing-EMC effect transition). Once more the nuclear effects are very dependent on the intrinsic $k_{T}$, EPS09 and EKS nPDFs have good agreement, as well as the dipole cross sections GBW, BUW, and DHJ (not shown in the graph). However, EPS08 and EPS09 disagree when shadowing is important, showing that the dilepton production can give hints about the shadowing intensity. At $y=$ -1.5 , nuclear effects of small $x_{2}$ drive EPS08 results to the lowest values obtained, and this is a direct result of the strong nuclear shadowing present at EPS08, that should be present at $\mathrm{LHC}$ even at negative rapidities.

As in RHIC, the color dipole approach and the IPM agree at high $p_{T} /\left\langle k_{T}\right\rangle$, however, at LHC energies, there is disagreement among the models, especially at mid rapidities. It is seen that the color dipole framework is less sensitive to the nuclear shadowing in the projectile than the IPM approach. Very little can be said about the color dipole cross section parametrizations, since they agree in 

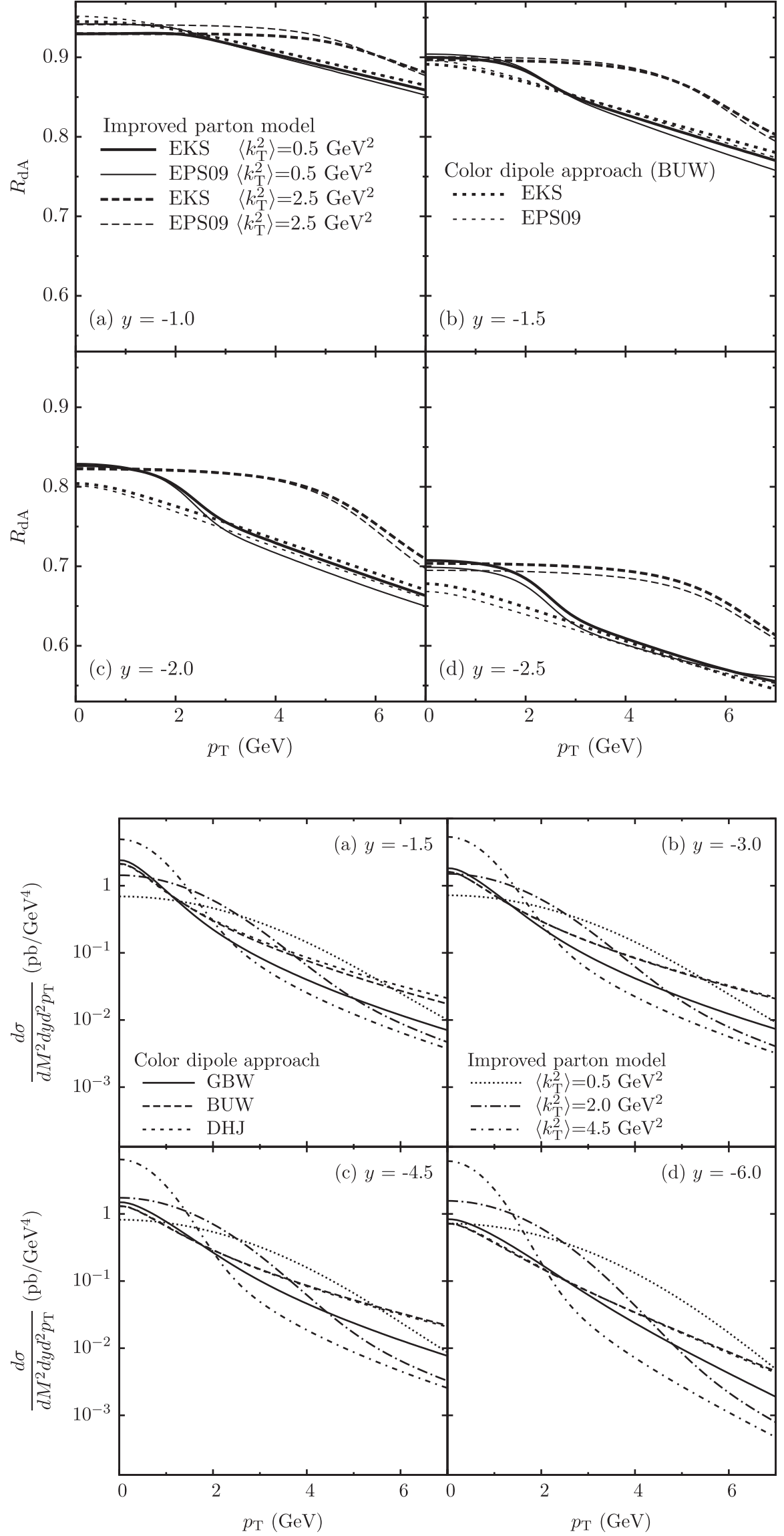

FIG. 5. Factor $R_{d A}$ at RHIC energies as a function of the transverse momentum at fixed rapidities, using EPS09 and EKS nPDFs.
FIG. 6. Dilepton production cross sections in $p-p$ collisions at LHC energies as a function of the transverse momentum at fixed rapidities. 


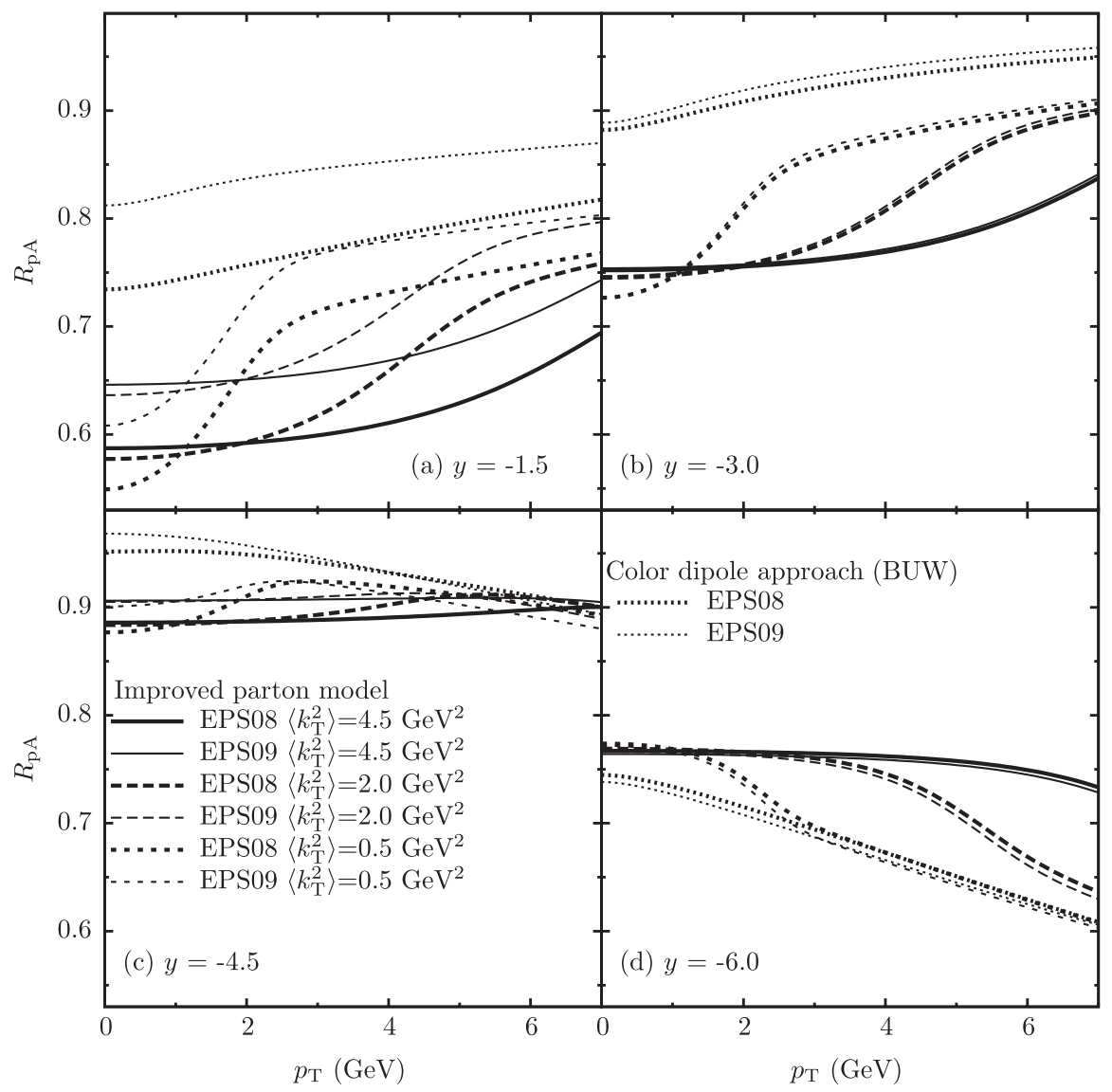

FIG. 7. Factor $R_{p A}$ at LHC energies as a function of the transverse momentum at fixed rapidities.

the entire range studied. That is not a surprise, since in the calculation of dilepton nuclear modification factors at backward rapidities the nuclear effects are not encoded in such parametrizations, but in the nPDF used. Concerning the $p_{T}$ slope, the results show that it is positive at more central rapidities and turns to negative at more backward rapidities. This is due to the fact that $x_{2}$ increases with $p_{T}$.
In Fig. 8, color dipole results obtained in Ref. [30] at forward rapidities considering the nucleus as a CGC phase compared to our results (EPS09 nPDF, BUW dipole cross section) at RHIC energies are shown. The three ways to calculate the dilepton production agree pretty well; even the transverse momentum dependence is the same. At forward rapidities, $R_{p A}$ increases with $p_{T}$, due to a related
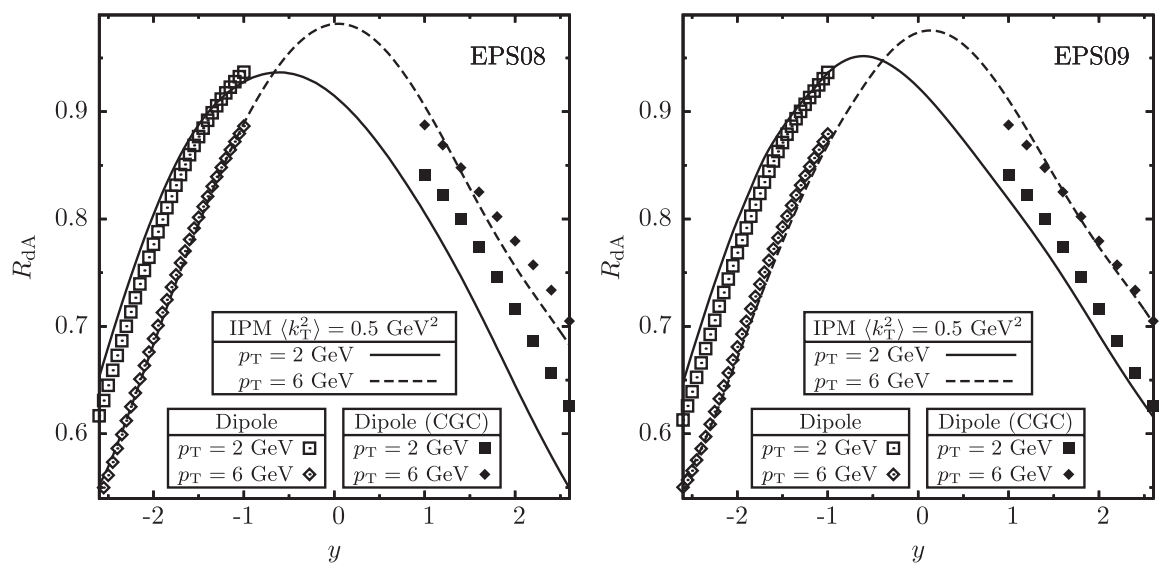

FIG. 8. Factor $R_{d A}$ calculated in the infinite momentum frame, in the color dipole picture at backward rapidities, and in the color dipole picture at forward rapidities considering the nucleus as a CGC [30] with EPS08 (left) and EPS09 (right) parametrizations at RHIC energies. 

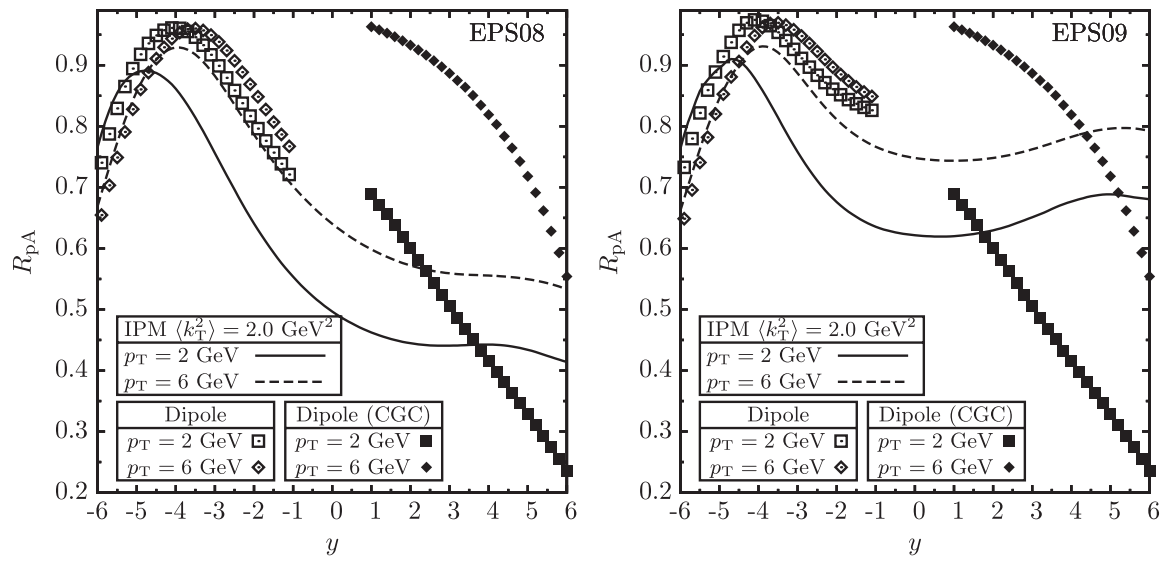

FIG. 9. Factor $R_{d A}$ calculated in the infinite momentum frame, in the color dipole picture at backward rapidities, and in the color dipole picture at forward rapidities considering the nucleus as a CGC [30] with EPS08 (left) and EPS09 (right) parametrizations at LHC energies.

increase in the parton momentum fraction, reducing the effect of shadowing. At backward rapidities, there is no shadowing but antishadowing effect, leading to a decrease of $R_{d A}$ with an increase of $p_{T}$.

While at LHC energies the same can be said about the qualitative transverse momentum dependence (Fig. 9), the results at forward rapidities disagree. In the CGC framework, only the gluon content of the target is taken into account. As long as the nuclear shadowing is considered, $R_{p A}$ will decrease as $x_{2}$ decreases. In the parton model, the two Compton subprocesses are dominant in the whole range of rapidity, due to the high small $x$ gluon distributions coming from both hadrons. At mid and forward rapidities, the main nuclear effect is the shadowing. At mid rapidities, gluons from both hadrons are equally important, so sea quark shadowing and gluon shadowing are taken into account. However, at forward rapidities, the projectile probes only the nuclear gluon distribution with its quarks. Hence, if both sea quark shadowing is stronger than gluon shadowing, and shadowing as an overall effect does not have a sufficiently steep dependence on $x$, the ratio $R_{p A}$ will increase from mid to forward rapidities at LHC, since one is progressively replacing quark shadowing by gluon shadowing. That is exactly what happens with the EPS09, which has an increase at forward rapidities as $y$ increases. In EPS08, shadowing is enhanced very fast as $x$ decreases in both gluon and quark distributions, therefore the nuclear modification factor always decreases for $y>0$.

\section{CONCLUSIONS}

The nuclear effects in the improved parton model and the color dipole approach through the nuclear modification factor were studied using the dilepton production as the observable at backward rapidities and RHIC and LHC energies. In the color dipole approach, nuclear effects could not discriminate among GBW, BUW, and DHJ models. Therefore, no hints about geometric scaling and the general behavior of dipole cross sections could be obtained.

The existence of intrinsic transverse momentum in the IPM can change $R_{p A}$ of $\approx 0.1$ when compared to color dipole approach results. Furthermore, the nuclear modification factor reflects the amount of intrinsic transverse momentum taken into account, being suitable to determine $\left\langle k_{T}^{2}\right\rangle$. These results suggest that the color dipole approach fails to include nonperturbative or higher order effects represented by the intrinsic $k_{T}$ and, most important, that these effects can be relevant when studying dilepton production. This renders the color dipole approach-as a parameterless description-unable to reproduce IPM results and possibly future dilepton data that agree with the IPM.

It was possible to see the effects of a strong shadowing such as the one introduced in EPS08 at $y<0$ at LHC energies. It follows that dilepton production at backward rapidities provides information about all nuclear effects at current energy levels and must be studied to complement the knowledge of forward rapidities.

At forward rapidities, we have compared our IPM results to earlier CGC results, still using nuclear modification ratios. At RHIC, different models qualitatively agree. At LHC, in the case of a not-so-strong shadowing, forward models qualitatively disagree due to the interplay of quark and of gluon shadowing. It is an important question whether the two kinds of models can reconcile their results either imposing a strong shadowing or reconsidering their quark contribution. In this sense, future LHC low-mass dilepton production data can be very elucidative and a restraining tool to the fits.

\section{ACKNOWLEDGMENTS}

This work was partially supported by $\mathrm{CNPq}$ and FAPERGS, Brazil. We thank M. A. Betemps for fruitful discussions. 
[1] R. Debbe, Nucl. Phys. A785, 76 (2007).

[2] D. d'Enterria, AIP Conf. Proc. 1038, 95 (2008).

[3] L. D. McLerran and R. Venugopalan, Phys. Rev. D 49, 3352 (1994).

[4] L. D. McLerran and R. Venugopalan, Phys. Rev. D49, 2233 (1994).

[5] T. Sakaguchi, J. Phys. G 35, 104025 (2008).

[6] I. Arsene et al. (BRAHMS), Phys. Rev. Lett. 93, 242303 (2004).

[7] M. A. Betemps, M. B. Gay Ducati, and E. G. de Oliveira, Phys. Rev. D 74, 094010 (2006).

[8] D. de Florian and R. Sassot, Phys. Rev. D 69, 074028 (2004).

[9] K. J. Eskola, V. J. Kolhinen, and P. V. Ruuskanen, Nucl. Phys. B535, 351 (1998).

[10] K. J. Eskola, V. J. Kolhinen, and C. A. Salgado, Eur. Phys. J. C 9, 61 (1999).

[11] K. J. Eskola, V. J. Kolhinen, H. Paukkunen, and C. A. Salgado, J. High Energy Phys. 05 (2007) 002.

[12] K. J. Golec-Biernat and M. Wusthoff, Phys. Rev. D 59, 014017 (1998).

[13] K. J. Eskola, H. Paukkunen, and C. A. Salgado, J. High Energy Phys. 07 (2008) 102.

[14] K. J. Eskola, H. Paukkunen, and C. A. Salgado, J. High Energy Phys. 04 (2009) 065.

[15] D. Boer, A. Utermann, and E. Wessels, Phys. Rev. D 77, 054014 (2008).

[16] A. Dumitru, A. Hayashigaki, and J. Jalilian-Marian, Nucl. Phys. A765, 464 (2006).
[17] A. Dumitru, A. Hayashigaki, and J. Jalilian-Marian, Nucl. Phys. A770, 57 (2006).

[18] H. Kowalski, L. Motyka, and G. Watt, Phys. Rev. D 74, 074016 (2006).

[19] G. Altarelli, G. Parisi, and R. Petronzio, Phys. Lett. 76B, 351 (1978).

[20] G. Altarelli, G. Parisi, and R. Petronzio, Phys. Lett. 76B, 356 (1978).

[21] B. Kopeliovich, in Workshop Hirschegg'95: Dynamical Properties of Hadrons in Nuclear Matter, edited by $\mathrm{H}$. Feldmeier and W. Nörenberg (GSI, Darmstadt, 1995), p. 102-112.

[22] J. Raufeisen, J.-C. Peng, and G. C. Nayak, Phys. Rev. D 66, 034024 (2002)

[23] S. D. Drell and T.-M. Yan, Phys. Rev. Lett. 25, 316 (1970).

[24] S. D. Drell and T.-M. Yan, Ann. Phys. (Leipzig) 66, 578 (1971).

[25] R. D. Field, Applications of Perturbative $Q C D$, Frontiers in Physics Vol. 77 (Addison-Wesley, Redwood City, 1989).

[26] P. Levai, G. Papp, G. G. Barnafoldi, and G. I. Fai, Eur. Phys. J. Special Topics 155, 89 (2008).

[27] B.Z. Kopeliovich, E. Levin, I. K. Potashnikova, and I. Schmidt, Phys. Rev. C 79, 064906 (2009).

[28] D. Stump et al., J. High Energy Phys. 10 (2003) 046.

[29] A. M. Stasto, K. J. Golec-Biernat, and J. Kwiecinski, Phys. Rev. Lett. 86, 596 (2001).

[30] M. A. Betemps and M. B. Gay Ducati, Phys. Rev. D 70, 116005 (2004). 